\title{
Alterations of DNA mismatch repair proteins and microsatellite instability levels in gastric cancer cell lines
}

\author{
Yuan Yao $^{1}$, Hong Tao ${ }^{1}$, Jae J Kim² ${ }^{2}$ Benjamin Burkhead ${ }^{1}$, Emilia Carloni ${ }^{3}$, \\ Antonio Gasbarrini ${ }^{3}$ and Antonia R Sepulveda ${ }^{1}$ \\ ${ }^{1}$ Department of Pathology, University of Pittsburgh, Pittsburgh, PA, USA; ${ }^{2}$ Division of Gastroenterology, \\ Samsung Medical Center, Seoul, Korea and ${ }^{3}$ Department of Medicine, Catholic University, Rome, Italy
}

\begin{abstract}
Alterations in DNA mismatch repair (MMR) proteins result in microsatellite instability (MSI), increased mutation accumulation at target genes and cancer development. About one-third of gastric cancers display high-level microsatellite instability (MSI-High) and low-level microsatellite instability (MSI-Low) is frequently detected. To determine whether variations in the levels of MMR proteins or mutations in the main DNA MMR genes are associated with MSI-Low and MSI-High in gastric cancer cell lines, the MSI status (MSI-High, MSI-Low or MSStable (MSS)) of $\mathbf{1 4}$ gastric cancer lines was determined using multiple clone analysis with a panel of five microsatellite markers. Protein levels of hMLH1, hMSH2, hMSH6, hPMS2 and hPMS1 were determined by Western blot. Sequence analysis of hMLH1 and hMSH2 was performed and the methylation status of the hMLH1 promoter was examined. The cell lines SNU1 and SNU638 showed MSI-High, decreased to essentially absent hMLH1 and hPMS2 and reduced hPMS1 and hMSH6 protein levels. The hMLH1 promoter region was hypermethylated in SNU638 cells. The MKN28, MKN87, KATOIII and SNU601 cell lines showed MSI-Low. The MMR protein levels of cells with MSI-Low status was similar to the levels detected in MSS cells. A marked decrease in the expression levels of MutL MMR proteins (hMLH1, hPMS2 and hPMS1) is associated with high levels of MSI mutations in gastric cancer cells. Gastric cancer cell lines with MSI-Low status do not show significant changes in the levels of the main DNA MMR proteins or mutations in the DNA mismatch repair genes hMSH2 and hMLH1. These well-characterized gastric cancer cell lines are a valuable resource to further our understanding of DNA MMR deficiency in cancer development, progression and prognosis.

Laboratory Investigation (2004) 84, 915-922, advance online publication, 10 May 2004; doi:10.1038/labinvest.3700117
\end{abstract}

Keywords: gastric cancer cell lines; microsatellite instability; mismatch repair genes; stomach cancer; mutations

The DNA mismatch repair (MMR) system is essential for replication fidelity, correcting DNA base mismatches left uncorrected by DNA polymerase. Efficient DNA mismatch repair requires the combined function of MutL (hMLH1-hPMS2 and hMLH1-hMLH3) and MutS (hMSH2-hMSH6 and hMSH2-hMSH3) heterodimers. ${ }^{1-5}$

In MMR-deficient cells, insertion or deletion of nucleotide bases at microsatellite sequences causing microsatellite instability (MSI)-type mutations occur at high frequency. ${ }^{1}$ Inactivation of genes by base insertion or deletion in small mononucleotide repeats in the coding region contributes to cancer

Correspondence: Dr AR Sepulveda, MD, PhD, Department of Pathology, University of Pittsburgh Medical Center, 200 Lothrop Street, PUH-A610, Pittsburgh, PA 15213, USA.

E-mail: sepulvedaar@upmc.edu

Received 3 February 2004; revised and accepted 30 March 2004; published online 10 May 2004 development and progression. ${ }^{6-11}$ MSI at multiple microsatellite loci occurs in a fraction of sporadic tumors, including gastric, endometrial and colorectal adenocarcinomas. ${ }^{12-14}$

A panel of five microsatellite loci including mono- and dinucleotide repeats, to test for microsatellite instability, was recommend by the NCI. ${ }^{15}$ This panel of markers allows for the classification of tumors into those with MSI-High level (MSI-High) when two or more loci $(30 \%$ or greater) show instability, MSI-Low level (MSI-Low) if only one locus shows MSI (less than 30\%), and microsatellite stable (MSS) if no instability is detected at the five microsatellite loci.

The MSI phenotype that occurs in tumors has been demonstrated to be caused by deficiency of one of the main DNA MMR proteins (hMLH1 and hMSH2) and in a smaller number of cases it is caused by deficiency of hMSH6, hMSH3 and hMLH3. ${ }^{16}$ Most sporadic cancers with MSI-High 
are characterized by loss of expression of hMLH1, secondary to methylation of CpG islands of the hMLH1 gene promoter, ${ }^{17,18}$ whereas, the MSI-High characteristic of hereditary nonpolyposis colorectal cancers (HNPCC) is caused by germline mutations in the human DNA MMR genes. ${ }^{16,19}$ The mechanisms underlying MSI-Low are not clear. ${ }^{20,21}$

Gastric cancer is the fourth most common cancer and the second leading cause of cancer mortality in the world. ${ }^{22-24} \mathrm{~A}$ subset of gastric carcinomas displaying the mutator phenotype and high-level MSI has been reported. In different studies, $15-30 \%$ of gastric carcinomas were reported to show MSI-High, one of the highest rates of MSI in sporadic cancers. ${ }^{12,14,25}$

Established gastric cancer cell lines provide a valuable tool to examine the molecular alterations of DNA MMR underlying MSI in gastric carcinomas. ${ }^{26,27}$ A comprehensive determination of MSIlevel and DNA MMR gene expression of gastric cancer cell lines is necessary before addressing the role of alterations of DNA MMR in the clinical behavior of gastric cancer cells and their response to therapy. In this study, we used a sensitive cell clone assay and a standard panel of microsatellite markers to characterize the microsatellite changes in 14 gastric cancer cell lines and determined the DNA MMR protein levels in each cell line. The methylation status of the hMLH1 promoter region was determined to evaluate whether hypermethylation is associated with loss of hMLH1 expression in gastric cancer cell lines. MSI-High, MSI-Low and MSS gastric cancer cell lines were identified in our study. The hMLH1 and hMSH2 cDNA sequences, which are the most often affected mismatch repair genes in MSI-High tumors, were sequenced to search for mutations or polymorphisms in their coding regions.

\section{Materials and methods}

\section{Gastric Cancer Cell Lines}

The gastric cell lines AGS (CRL-1739), KATOIII (HBT-103), N87 (CRL-5822), SNU1 (CRL-5971), SNU601 (CRL-5974) and SNU16 were obtained from the ATCC, Manassas, VA, USA. The cell lines SNU638, SNU668 and SNU719 were previously reported..$^{3,27}$ The cell lines MKN1, MKN7, MKN28, MKN74, GTL16 and TMK1, were kindly given by Dr Richard Hamelin and were previously reported. ${ }^{28,29}$ The cells were grown in RPMI-1640 medium containing 10\% fetal bovine serum, penicillin and streptomycin (GIBCO BRL, Gaithersburgh, MD, USA), except KATOIII cells that were grown in RPMI-1640 containing $20 \%$ fetal bovine serum and penicillin and streptomycin.

\section{MSI Analysis}

Each cell line was cultured to obtain individual cell clones by serial dilutions. Genomic DNA was extracted from 10 separate cell clones per marker examined, as previously described. ${ }^{3}$ The microsatellite markers included the BAT25 and BAT26 mononucleotide markers and the D2S123, D17S250, D13S170 dinucleotide repeat markers. ${ }^{3,15}$ The oligonucleotide primers were purchased from Research Genetics, Inc. Huntsville, AL, USA. One of the primers was end-labeled with $\gamma^{32} \mathrm{P}$-ATP using T4 polynucleotide kinase (Promega Corp., Madison, WI, USA). PCR amplifications were performed for 35 cycles, consisting of $1 \mathrm{~min}$ at $94^{\circ} \mathrm{C}, 1 \mathrm{~min}$ at $50^{\circ} \mathrm{C}$, and $1 \mathrm{~min}$ at $72^{\circ} \mathrm{C}$, as described before. ${ }^{3}$ The amplified products were separated through $6 \%$ acrylamide, $5.6 \mathrm{M}$ urea sequencing gels. A microsatellite marker was scored as MSI-positive when there were two or more different allele patterns for the microsatellite locus in the multiple cell clones of the same gastric cell line. Cell lines were scored as having MSI-High if more than $30 \%$ of the examined markers were MSI-positive (two or more markers out of five). Cell lines were considered to be MSS if no unstable microsatellites were found and were considered to have low-frequency MSI (MSI-Low), when MSI was present in less than $30 \%$ of the markers (one marker out of five). ${ }^{15,30}$

\section{Western Blot Analysis}

Cells were cultured to $90 \%$ confluence in $10 \mathrm{~cm}$ plates, and protein extracts were prepared in Laemmli sample buffer. ${ }^{3}$ Total protein $(20 \mu \mathrm{g})$ were resolved through SDS-PAGE gel electrophoresis and transferred to an Immobilon-P membrane (Millipore, Bedford, MA, USA). The membranes were incubated for $1 \mathrm{~h}$ at room temperature with each individual primary antibody. The antibodies used were the mouse monoclonal anti-hMLH1 (C20, clone G168-15; Pharmingen, San Diego, CA, USA), rabbit polyclonal anti-hPMS1 (C-20; Santa Cruz Biotechnology; Santa Cruz, CA, USA), rabbit polyclonal anti-hPMS2 (C-20; Santa Cruz Biotechnology), mouse monoclonal anti-hMSH2 (Clone AB-3 FE11; Oncogene Research, Cambridge, MA, USA), polyclonal goat anti-hMSH6 (N-20; Santa Cruz Biotechnology), and mouse monoclonal anti- $\beta$-actin (MAB1501; Chemicon International, Temecula, CA, USA). Antibodies were used diluted at 1:1000. After incubation with each primary antibody, the membrane was incubated with goat anti-rabbit IgG-HRP or goat anti-mouse IgG-HRP (Santa Cruz Biotechnology, Santa Cruz, CA, USA) for $1 \mathrm{~h}$ at room temperature. The proteins were then detected by an ECL plus Western blotting detection system (Amersham Biosciences Corp, Piscataway, NJ, USA) and exposed to X-ray film. Quantification was performed using a chemiluminescence detec- 
tion system with the Quantity One version 4.2.2 software (ChemiDoc, Bio-Rad, Hercules, CA, USA). All results were confirmed by repeat experiments.

\section{Immunohistochemical Stains}

Immunohistochemical stains were performed using formalin-fixed, paraffin-embedded tissue sections of cell blocks containing individual cell lines. Antigen retrieval was performed by steaming the samples in $0.1 \mathrm{M}$ citric acid at $\mathrm{pH} 6.0$ for $20 \mathrm{~min}$. Negative controls were stained without adding primary antibody. The antibodies used were the mouse monoclonal anti-hMLH1 clone 13291A (Santa Cruz Biotechnology, Santa Cruz, CA, USA), and the mouse monoclonal anti-hMSH2 (Clone AB-3 FE11; Oncogene Research, Cambridge, MA, USA), at 1:800 and 1:200 dilutions, respectively, and were incubated with the tissue sections overnight at $4^{\circ} \mathrm{C}$. Immunoreactions were detected with a standard avidin-biotin detection system using the LSAB, HRP kit (DAKO, CA, USA), in a DAKO autostainer.

\section{Promoter Methylation Assays}

The hMLH1 promoter methylation was analyzed as described by Kane et al. ${ }^{31}$ Genomic DNA samples were extracted from the gastric cancer cell lines SNU638, MKN74, MKN28, KATOIII, N87, SNU601, MKN1, SNU1, AGS, MKN7, SNU5, SNU601, SNU668, SNU719, GTL16 and TMK1. DNA samples (250 ng) were digested with $75 \mathrm{U}$ of HpaII restriction endonuclease (New England Biolabs) in a $20 \mu \mathrm{l}$ reaction volume for $6 \mathrm{~h}$ at $37^{\circ} \mathrm{C}$. To analyze the cleavage of the hMLH1 promoter region, $1 \mu \mathrm{l}$ of each HpaII digest was amplified by PCR in $25 \mu \mathrm{l}$ reactions containing $2.5 \mu \mathrm{l}$ of $10 \times$ PCR reaction buffer II and $1.5 \mu \mathrm{l}$ of $25 \mathrm{mM} \mathrm{MgCl}$ (Applied Biosystems, Foster City, CA, USA), $0.4 \mu \mathrm{M}$ of each of the four deoxynucleotide triphosphates, $1.5 \mathrm{U}$ of Taq Gold polymerase (Applied Biosystems, Foster City, CA, USA), and 25 pmol of each primer (5'-CGCTCGTAGTAT TCGTGC- $3^{\prime}$ and $5^{\prime}$-TCAGTGCCTCGTGCTCAC- $3^{\prime}$ ), designed to amplify nucleotides -670 to -67 of hMLH1 (Genebank Accession No. U83845). PCR reactions were performed for one cycle at $95^{\circ} \mathrm{C}$ for $5 \mathrm{~min}$, followed by 30 cycles at $94^{\circ} \mathrm{C}$ for $30 \mathrm{~s}, 55^{\circ} \mathrm{C}$ for $30 \mathrm{~s}$, and $72^{\circ} \mathrm{C}$ for $1 \mathrm{~min}$, followed by one cycle at $72^{\circ} \mathrm{C}$ for $7 \mathrm{~min}$. Negative controls included amplification in the absence of DNA and an unrelated plasmid (pcDNA3.1CA13-EGFP) was used for DNA control. The resulting amplification products were analyzed by $2 \%$ agarose gel electrophoresis.

\section{Sequence Analysis}

Total RNA was extracted from the cell lines MKN87, KATOIII and MKN28 with RNAwiz (Ambion, Austin, TX, USA). The following primer pairs were designed to sequence the cDNA regions of hMLH1 (MLH1.1 F-gccgccgggatccatgtcgttggcagg; MLH1.1Rcggcggccctaggtgtatcggaatacagag; MLH1.2 F-ccgggat caggaaagaagat; MLH1.2 R-aatgcctgcattgttactga; MLH1.3 F-caacatagccacgaggagaa; MLH1.3 Rgaatgggtgtgtgtttttgg; MLH1.4 F-cttcatcaaccatcgtctgg; MLH1.4 R-cgggaatctgtacgaaccatc; MLH1.5 Fcctctgggg agatggttaaa; MLH1.5 R-ctttcgggaatcatcttc ca; MLH1.6 F-agcaaccccagaaagagaca; MLH1.6 Rttcctctgtccagccactct; MLH1.7 F-aggttatcggagccagcac; MLH1.7 R-ctgcttccggatggaataga) and hMSH2 (MSH2.1 F-accaggaggtgaggaggttt; MSH2.1 R-gattgc caggagaagcctta; MSH2.2 F-gct ggaaataaggcatccaa; MSH2.2 R-tgtcttttgtggaaaagtcagc; MSH2.3 F-aga catggggaaactgagaca; MSH2.3 R-tctttgtccttgaggggtttt; MSH2.4 F-gagcccttaacctttttcagg; MSH2.4 R-ggagtca caaaa actgccaac; MSH2.5 F-ttgttaccgactctatcagggta; MSH2.5 R-ttgttacga aggactttttcttcc; MSH2.6 F-ccaga gatcttggcttggac; MSH2.6 R-ttcttccttgtcctttctcca; MSH2.7 F-gatgctgttgtcagctttgc; MSH2.7 R- ttccaa catttcagccatga; MSH2.8 F-gtgtccattgtggactgcat; MSH2.8 R-agcaagctctgc aacatgaa).

Total RNA $(2.5 \mu \mathrm{g})$ were used for reverse transcription with random hexamers ( $50 \mathrm{ng}$ ), $500 \mu \mathrm{M}$ dNTPs, $0.1 \mathrm{mM}$ DTT and $100 \mathrm{U}$ of Superscript II enzyme in a $20 \mu \mathrm{l}$ reaction mixture, containing $1 \mathrm{X}$ First-Strand Buffer (Invitrogen) and $10 \mathrm{U}$ of RNase Inhibitor. A $2 \mu \mathrm{l}$ aliquot of each cDNA was amplified in a $50 \mu \mathrm{l}$ reaction containing 1X PCR buffer II, $200 \mu \mathrm{M}$ dNTPs, $1.5 \mathrm{mM} \mathrm{MgCl}_{2}, 50 \mathrm{pmol}$ of forward and reverse primers, and $2 \mathrm{U}$ of AmpliTaq Gold enzyme (Applied Biosystems, Branchburg, NJ, USA). The cDNA fragments were cloned into the pSTBlue-1 Blunt Vector (Novagen, San Diego, CA, USA). The ligation products were transformed into Nova Blue competent bacterial cells (Novagen, San Diego, CA, USA), and individual bacterial clones were isolated for plasmid preparation. At least two separate bacterial clones were picked for each cloned fragment and individually sequenced using a T7 promoter oligonucleotide primer present in the pSTBlue-1 vector. Automated sequence analyses were performed at the DNA sequencing facility of the University of Pittsburgh.

\section{Data Analysis}

The significance of altered levels of MMR proteins in cells with different MSI levels was determined by a Student's $t$-test (two-tailed) using the SigmaStat 3.0. (SPSS, Inc.). Significance was defined as a $P$ value $<0.05$.

\section{Results}

\section{Microsatellite Instability Status of Gastric Cancer Cell Lines}

The MSI status of 14 established gastric cancer cell lines (AGS, N87, KATOIII, SNU1, SNU16, 
SNU601, SNU638, SNU668, SNU719, MKN1, MKN7, MKN28, GTL16 and TMK1, was determined by PCR amplification of a set of five microsatellite markers, following the NCI-recommendations for MSI testing. ${ }^{12,15}$ The evaluation of MSI in a cell line using individual cell clones is more sensitive for MSI detection than the amplification of DNA from the whole cell culture.,32 Therefore, MSI at a given microsatellite locus was detected by comparison of the allele patterns of multiple separate cell clones of each cell line, as previously described. ${ }^{3,33-35}$ The multiple cell clones of each gastric cancer cell line were obtained by serial dilutions and plating in 96-microwell plates. At least 10 separate cell clones were picked for each cell line for each marker, and overall, approximately 600 individual clones were subjected to PCR amplification. The numbers of different allele patterns for each microsatellite marker in a given cell line was scored. If different alleles of a given microsatellite marker were detected in the separate cell clones of a given cell line, then that locus was scored as MSI-positive. When two or more of the five microsatellite markers showed MSI, based on the NCI criteria, ${ }^{15}$ MSI-High was scored. If only one of the five markers showed MSI, this was scored as MSILow, and if none of the five markers showed instability, the cell line was considered to display a MSS phenotype. For example, the cell line SNU638 displayed four different alleles characterized by different size in the eight clones that yielded positive amplification with the BAT26 marker, thus showing MSI at the BAT26 locus. The SNU638 cell line also showed MSI at the BAT25 locus, meeting the criteria for MSI-High (Figure 1 and Table 1). The SNU1 cell line also showed MSI-High level, with instability at the BAT26, D2S123 and D13S170 loci. The cell lines MKN28, MKN87, SNU601 and KATOIII showed low-level MSI with instability at either the BAT26 or D17S250 locus (Figure 1 and Table 1). The remaining eight gastric cancer cell lines tested were MSS. The BAT26 marker, right which has been recognized as an excellent marker of $\mathrm{MSI}^{36}$ was the most often altered, showing instability in four of the six lines with MSI. Interestingly, BAT26 showed MSI both in cell lines with MSI-High and in cells with MSI-Low.

\section{Alteration in Mismatch Protein Repair Levels Underlying MSI in Gastric Cancer Cells}

The levels of the MutL (hMLH1, hPMS1, hPMS2) and MutS (hMSH2 and hMSH6) mismatch repair



b

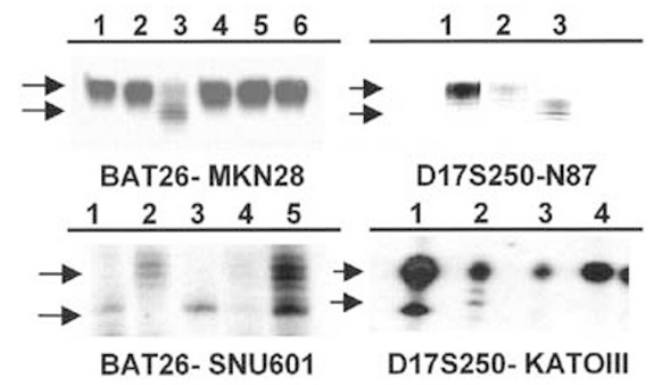

Figure 1 MSI in gastric cancer cell lines. Separate cell clones from each cell line were used for PCR amplification with the oligonucleotide primer set specific for each microsatellite locus. Up to 10 separate cell clones of each cell line were tested. (a) MSIhigh characterizes the gastric cells lines SNU638 and SNU1. The microsatellite markers BAT26 and BAT25 display different allele patterns in separate clones of the SNU638 cell line. The microsatellite markers BAT26, D2S123 and D13S170 show different alleles in separate clones of the SNU1 cell line. (b) MSI-Low in the cell lines MKN28, MKN87, SNU601 and KATOIII was detected at the BAT26 and D17S250 loci. The arrows indicate the presence of different amplification fragments of separate cell clones of each cell line, characteristic of microsatellite instability.

Table 1 MSI status of MSI-positive gastric cancer cell lines

\begin{tabular}{llcccc}
\hline Cell line & MSI level & BAT26 & \multirow{2}{*}{ BAT25 } & D2S123 & D13S170 \\
\hline SNU638 & MSI-High & MSI & MSI & MSS & MSS \\
SNU1 & MSI-High & MSI & MSS & MSI & MSI \\
MKN28 & MSI-Low & MSI & MSS & MSS & MSS \\
N87 & MSI-Low & MSS & MSS & MSS & MSS \\
SNU601 & MSI-Low & MSI & MSS & MSS \\
Kato III & MSI-Low & MSS & MSS & MSS & MSS \\
\end{tabular}

The microsatellite loci that showed instability (MSI) or that were stable (MSS) are indicated. 
proteins were determined by Western blot analysis and immunostains. The two cell lines with MSIHigh (SNU1 and SNU638) showed absence of hMLH1 and hPMS2 and reduced levels of hPMS1, as shown in a representative Western blot (Figure 2). In addition to an absence of MutL complex, the SNU638 also showed a decreased level of hMSH6, presumably resulting in a combined defect of MutL and MutS mismatch repair complex (Figure 3). The cell lines MKN28, N87, SNU601 and KATOIII, characterized by MSI-Low did not show significant changes in the levels of MutL or MutS mismatch repair proteins, as compared to the levels of protein expression in the MSS cell lines (Figures 2 and 3).

The absence of hMLH1 in the SNU1 and SNU638 cells was also confirmed by immunohistochemical stain of sections of cell blocks (Figure 4), while the other cell lines expressed normal levels of hMLH1 and hMSH2 proteins with a normal nuclear localization pattern (Figure 4).

\section{Sequence Analysis of the Main DNA MMR Genes in MSI-Low Gastric Cancer Cell Lines}

Since no alterations in the levels of MMR proteins were seen in MSI-Low cells, we sequenced the coding regions of the main DNA mismatch repair genes hMSH2 and hMLH1, to determine whether mutations were associated with the MSI-Low phenotype. Several sets of nucleotide primers were designed to amplify the entire coding regions of hMSH2 and hMLH1 and were used for PCR amplification from genomic DNA extracted from the MSI-Low cells (SNU601, MKN28, N87 and KATOIII). The PCR fragments were subcloned into the pSTBlue-1 vector and clones containing the separately amplified DNA fragments were sequenced. Plasmid DNA from at least two bacterial clones of each DNA fragment was sequenced on

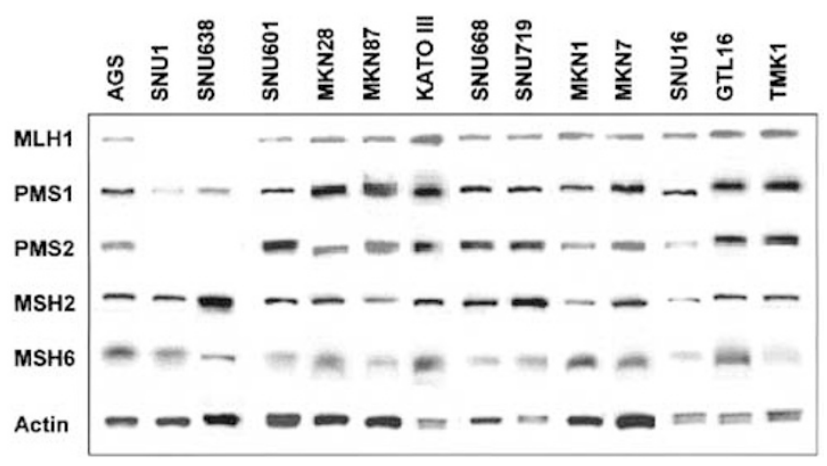

Figure 2 Mismatch repair protein levels in gastric cancer cell lines. Western blot with specific antibodies against hMLH1, hPMS1, hPMS2, hMSH2, hMSH6 and actin were performed with the gastric cancer cell lines: AGS, SNU1, SNU638, SNU601, MKN28, MKN87, KATOIII, SNU668, SNU719, MKN1, MKN7, SNU16, GTL16 and TMK1. The cell lines SNU1 and SNU638 show absence of hMLH1 and hPMS2 and decreased levels of hPMS1. both DNA strands. The hMLH1 gene in MKN28 cells was characterized by an A to $G$ transition polymorphism resulting in an isoleucine to valine change at codon 655. No other mutations were identified in the entire coding regions of hMLH1 or hMSH2 in the MSI-Low cell lines.

\section{Analysis of Methylation Status of the hMLH1 Promoter in Gastric Cancer Cell Lines with MSI-High and MSI-Low}

The absence of hMLH1 protein in sporadic cancers displaying MSI-High has been shown to be related to silencing of gene transcription by methylation of the hMLH1 promoter region. In contrast, in HNPCC with typical MSI-High, the absence of hMLH1 proteins is secondary to point mutations in the coding region or allelic loss. ${ }^{37}$ The SNU1 cell line has been shown to carry a nonsense point mutation
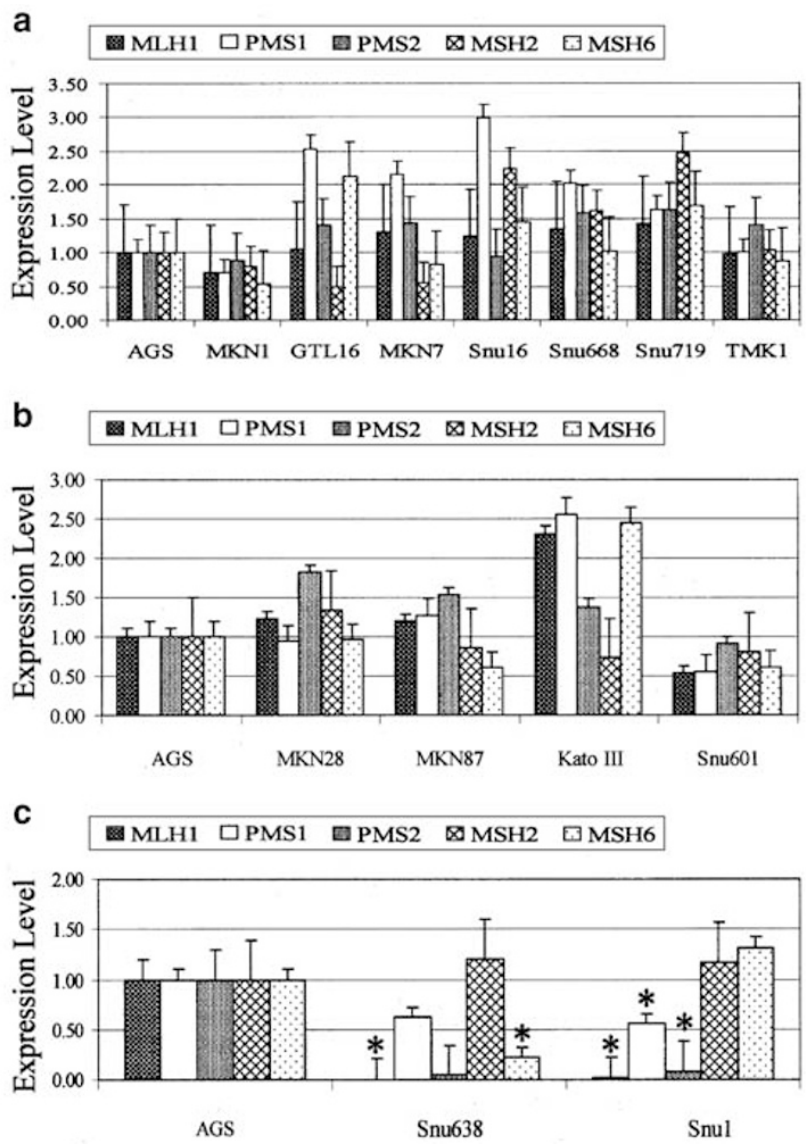

Figure 3 Expression patterns of MMR proteins in gastric cancer cell lines. (a) MMR protein expression levels in MSS gastric cancer cell lines. (b) MMR protein expression levels in gastric cancer cell lines with MSI-Low. (c) MMR protein expression levels in gastric cancer cells with MSI-High. * Indicates the statistically significant changes in protein levels in MSI-High cell lines, compared to the levels of the proteins in mismatch repair competent MSI-negative AGS cells. In SNU1 cells hMLH1 $P<0.003$, hPMS2 $P=0.02$, and hPMS1 $P=0.039$. In SNU638 cells hMLH1 $P<0.001$, hPMS2 $=0.073$ and hMSH6 $<0.05$. 

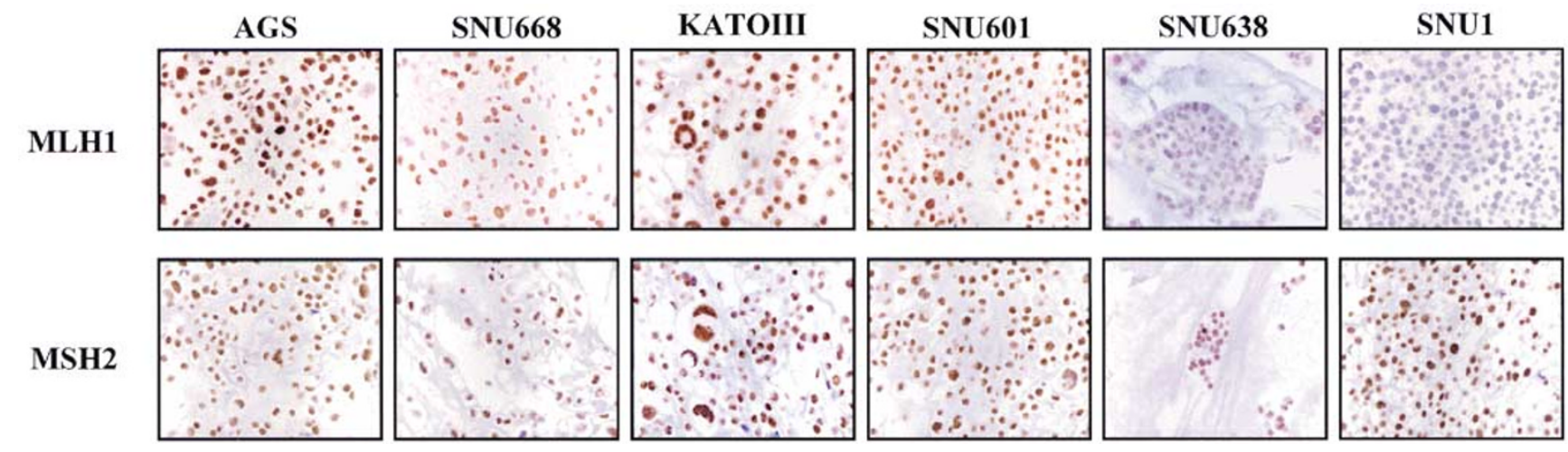

Figure 4 Immunohistochemical analyses of hMLH1 and hMHS2 expression in gastric cancer cell lines. The hMLH1 and hMSH2 genes were expressed at detectable levels in the nucleus of gastric cancer cell lines. Only the SNU638 and SNU1 cell lines did not express hMLH1. All cell lines expressed detectable levels of hMSH2 protein.

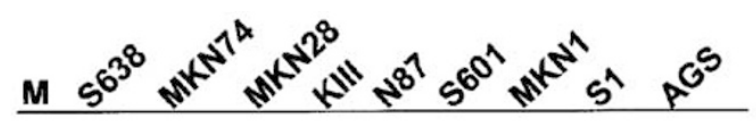

UHUHUHUHUHUHUHUH UH

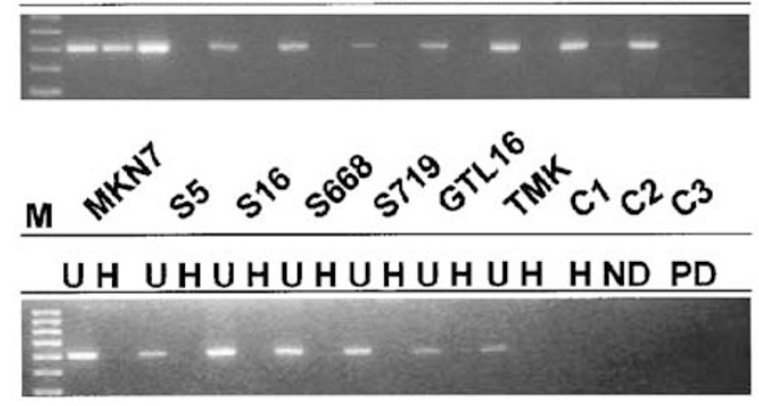

Figure 5 Analysis of methylation status of the hMLH1 promoter region. Amplification of the hMLH1 promoter region from the gastric cancer cell lines SNU638, MKN74, MKN28, KATOIII, MKN87, SNU601, MKN1, SNU1, AGS, MKN7, SNU5, SNU601, SNU668, SNU719, GTL16 and TMK1, after digestion of genomic DNA by HpaII (H) or without digestion (U). Negative controls include: (C1) plasmid DNA digested with HpaII, (C2) amplification of reaction without DNA and (C3) amplification of plasmid DNA without previous HpaII digestion. Amplification of the promoter region A after HpaII digestion was only observed in SNU638 cells, indicating methylation of the hMLH1 promoter region of this cell line.

at codon $226,{ }^{38,39}$ while the SNU638 cell line was shown to display methylation of the hMLH1 promoter region. ${ }^{40}$ However, it is not known whether the loss of hMLH1 expression results from methylation of the promoter region or from mutation or allelic loss of the second hMLH1 allele. Therefore, we determined the methylation status of hMLH1 in MSI-Low cells and in the MSI-High SNU1 and SNU 638 cells to assess whether there was an association with the MSI status. The methylation status of the hMLH1 promoter was determined by PCR after digestion of genomic DNA by the methylation-sensitive HpaII restriction enzyme as previously reported. ${ }^{31}$ Only the SNU638 cell line showed hMLH1 promoter methylation.
MSS cell lines and the MSI-Low lines did not show methylation of the hMLH1 promoter region (Figure 5). Interestingly, the MSI-High SNU1 did not show hMLH1 promoter methylation. Thus, given previous studies indicating that SNU1 cells display a truncating mutation at codon $226,{ }^{38}$ our data suggest that the second hMLH1 allele is probably inactivated by a mutation or allelic loss.

\section{Discussion}

Gastric carcinomas are among the tumors that most frequently display MSI. ${ }^{12,14,25}$ The relationship between MSI pattern and variation in the levels of DNA MMR proteins of the MutS and MutL complex in gastric cancer is not well understood. It is not known whether MSI-Low is associated with reduced levels of any of the known DNA MMR proteins. Further, the role of hMSH2 deficiency underlying gastric cancer with MSI is not well characterized.

In this study, we used 14 established gastric carcinoma cell lines to characterize the patterns of MSI and the underlying DNA mismatch repair protein expression levels. Our studies demonstrate that the gastric cancer cell lines SNU1 and SNU638 show characteristic MSI-High, the cell lines SNU601, N87, MKN28 and KATOIII, show MSILow while the remaining eight gastric cancer cell lines are MSS. The SNU1 and S638 cell lines display the MSI-High phenotype, showing lack of expression of hMLH1, a secondary loss of hPMS2, and a drop in hPMS1 levels. No alterations were found in the hMSH2 gene and protein levels. However, the SNU638 cells showed reduced levels of hMSH6 in the presence of normal levels of hMSH2. It is not clear why hMSH6 levels are decreased in SNU 638 cells, however, it is possible that hMSH6 might have undergone truncation mutations resulting in unstable proteins, since hMSH6 is a known target of MSI-type mutagenesis, and might have undergone mutation secondary to the severe deficiency of MutL in these cells. 
Sequence analysis of hMSH2 and hMLH1 from gastric cell lines showing MSI-Low revealed an A to $\mathrm{G}$ transition polymorphism in the hMLH1 gene in MKN28 cells, resulting in an isoleucine to valine change at codon 655. No other mutations were identified in the entire coding regions of hMLH1 or hMSH2 in these cells. Thus, it appears unlikely that hMLH1 or hMSH2 mutations are responsible for the MSI-Low phenotype in these cells. The cell lines MKN28, N87, SNU601 and KATOIII, characterized by MSI-Low did not show significant changes in the levels of MutL or MutS mismatch repair proteins, as compared to the levels of protein expression in the MSS cell lines, suggesting that absence or significantly reduced levels of the main MMR proteins are not the underlying mechanism for the MSI-Low phenotype. Nonetheless, we hypothesize that MSILow might be critical for cancer development by facilitating gene mutations, possibly associated with a relative deficiency of critical levels of MMR complexes during the cell cycle in cells with increased cell turnover. For example, it has been shown that the MMR system is necessary for G2 arrest of the cell cycle or cell death in cells with alkylation damage. ${ }^{41}$ The importance of an appropriate period of $\mathrm{G} 2$ arrest is that during this checkpoint, repair of damaged DNA occurs, preventing the replication of mutated DNA. If this mechanism is impaired, MSI-Low might result in the inactivation of regulatory genes, such as the transforming growth factor $\beta$-receptor type II, and other known target genes of microsatellite-type mutations. ${ }^{9,10,42}$ Therefore, MMR defects, as manifested by MSI-Low, could be important in early stages of gastric carcinogenesis, despite the fact that changes in MMR protein levels might not be apparent in cancer cells with the MSI-Low phenotype.

In summary, in this report we provide a detailed characterization of the DNA mismatch repair system and MSI of 14 established gastric cancer cell lines. These cells represent a very useful tool for future experimental studies addressing the role of DNA mismatch repair in the biological behavior of gastric cancer.

\section{Acknowledgement}

This study was supported by research project Grant RPG-00-230-01-CNE from the American Cancer Society (ARS).

\section{References}

1 Kolodner RD, Marsischky GT. Eukaryotic DNA mismatch repair. Curr Opin Genet Dev 1999;9:89-96.

2 Lipkin SM, Wang V, Jacoby R, et al. MLH3: a DNA mismatch repair gene associated with mammalian microsatellite instability. Nat Genet 2000;24:27-35.
3 Leung WK, Kim JJ, Wu L, et al. Identification of a second MutL DNA mismatch repair complex (hPMS1 and hMLH1) in human epithelial cells. J Biol Chem 2000;275:15728-15732.

4 Liu T, Yan H, Kuismanen S, et al. The role of hPMS1 and hPMS2 in predisposing to colorectal cancer. Cancer Res 2001;61:7798-7802.

5 Raschle M, Marra G, Nystrom-Lahti M, et al. Identification of hMutLbeta, a heterodimer of hMLH1 and hPMS1. J Biol Chem 1999;274:32368-32375.

6 Park K, Kim SJ, Bang YJ, et al. Genetic changes in the transforming growth factor beta (TGF-beta) type II receptor gene in human gastric cancer cells: correlation with sensitivity to growth inhibition by TGF-beta. Proc Natl Acad Sci USA 1994;91: 8772-8776.

7 Oliveira C, Seruca R, Seixas M, et al. The clinicopathological features of gastric carcinomas with microsatellite instability may be mediated by mutations of different "target genes": a study of the TGFbeta RII, IGFII R, and BAX genes. Am J Pathol 1998;153: 1211-1219.

8 Abe T, Ouyang H, Migita T, et al. The somatic mutation frequency of the transforming growth factor beta receptor type II gene varies widely among different cancers with microsatellite instability. Eur J Surg Oncol 1996;22:474-477.

9 Myeroff LL, Parsons R, Kim SJ, et al. A transforming growth factor beta receptor type II gene mutation common in colon and gastric but rare in endometrial cancers with microsatellite instability. Cancer Res 1995;55:5545-5547.

10 Ohue M, Tomita N, Monden T, et al. Mutations of the transforming growth factor beta type II receptor gene and microsatellite instability in gastric cancer. Int J Cancer 1996;68:203-206.

11 Ottini L, Falchetti M, D’Amico C, et al. Mutations at coding mononucleotide repeats in gastric cancer with the microsatellite mutator phenotype. Oncogene 1998; 16:2767-2772.

12 Leung WK, Kim JJ, Kim JG, et al. Microsatellite instability in gastric intestinal metaplasia in patients with and without gastric cancer. Am J Pathol 2000; 156:537-543.

13 Arzimanoglou II, Gilbert F, Barber HR. Microsatellite instability in human solid tumors. Cancer 1998;82: 1808-1820.

14 Duval A, Hamelin R. Genetic instability in human mismatch repair deficient cancers. Ann Genet 2002; 45:71-75.

15 Boland CR, Thibodeau SN, Hamilton SR, et al. A National Cancer Institute Workshop on Microsatellite Instability for cancer detection and familial predisposition: development of international criteria for the determination of microsatellite instability in colorectal cancer. Cancer Res 1998;58:5248-5257.

16 Boland CR. Molecular genetics of hereditary nonpolyposis colorectal cancer. Ann NY Acad Sci 2000;910:50-59. discussion 59-61.

17 Fleisher AS, Esteller M, Wang S, et al. Hypermethylation of the hMLH1 gene promoter in human gastric cancers with microsatellite instability. Cancer Res 1999;59:1090-1095.

18 Herman JG, Umar A, Polyak K, et al. Incidence and functional consequences of hMLH1 promoter hypermethylation in colorectal carcinoma. Proc Natl Acad Sci USA 1998;95:6870-6875. 
19 Liu B, Parsons R, Papadopoulos N, et al. Analysis of mismatch repair genes in hereditary nonpolyposis colorectal cancer patients. Nat Med 1996; 2:169-174.

20 Tomlinson I, Halford S, Aaltonen L, et al. Does MSIlow exist? J Pathol 2002;197:6-13.

21 Pinto M, Oliveira C, Machado JC, et al. MSI-L gastric carcinomas share the hMLH1 methylation status of MSI-H carcinomas but not their clinicopathological profile. Lab Invest 2000;80:1915-1923.

22 Ferlay JBF, Pisani P, Parkin DM. GLOBOCAN 2000: Cancer Incidence, Mortality, and Prevalence Worldwide. Version: 1.0 ed. International Agency for Research on Cancer Press, 2001.

23 Parkin DM, Bray F, Ferlay J, et al. Estimating the world cancer burden: Globocan 2000. Int J Cancer 2001;94:153-156.

24 Jemal A, Thomas A, Murray T, et al. Cancer statistics, 2002. CA Cancer J Clin 2002;52:23-47.

25 Semba S, Yokozaki H, Yamamoto S, et al. Microsatellite instability in precancerous lesions and adenocarcinomas of the stomach. Cancer 1996;77(Suppl 8): 1620-1627.

26 Bae SI, Park JG, Kim YI, et al. Genetic alterations in gastric cancer cell lines and their original tissues. Int J Cancer 2000;87:512-516.

27 Park JG, Yang HK, Kim WH, et al. Establishment and characterization of human gastric carcinoma cell lines. Int J Cancer 1997;70:443-449.

28 Yokozaki H. Molecular characteristics of eight gastric cancer cell lines established in Japan. Pathol Int 2000;50:767-777.

29 Ochiai A, Yasui W, Tahara E. Growth-promoting effect of gastrin on human gastric carcinoma cell line TMK-1. Jpn J Cancer Res 1985;76:1064-1071.

30 Dietmaier W, Wallinger S, Bocker T, et al. Diagnostic microsatellite instability: definition and correlation with mismatch repair protein expression. Cancer Res 1997;57:4749-4756.

31 Kane M, Loda M, Gaida G, et al. Methylation of the hMLH1 promoter correlates with lack of expression of hMLH1 in sporadic colon tumors and mismatch-repair defective human tumor cell lines. Cancer Res 1997; 57:808-811.

32 Liu B, Parsons RE, Hamilton SR, et al. hMSH2 mutations in hereditary nonpolyposis colorectal cancer kindreds. Cancer Res 1994;54:4590-4594.

33 Umar A, Boyer JC, Thomas DC, et al. Defective mismatch repair in extracts of colorectal and endometrial cancer cell lines exhibiting microsatellite instability. J Biol Chem 1994;269:14367-14370.

34 Parsons R, Guo-Min L, Longley M, et al. Mismatch repair deficiency in phenotypically normal human cells. Science 1995;268:738-740.

35 Reitmair AH, Risley R, Bristow RG, et al. Mutator phenotype in Msh2-deficient murine embryonic fibroblasts. Cancer Research 1997;57:3765-3771.

36 Hoang JM, Cottu PH, Thuille B, et al. BAT-26, an indicator of the replication error phenotype in colorectal cancers and cell lines. Cancer Res 1997;57: 300-303.

37 Wheeler JM, Loukola A, Aaltonen LA, et al. The role of hypermethylation of the hMLH1 promoter region in HNPCC versus MSI+ sporadic colorectal cancers. J Med Genet 2000;37:588-592.

38 Shin K, Yang Y, Park J. Absence or decreased levels of the hMLH1 protein in human gastric carcinoma cell lines: implication of hMLH1 in alkylation tolerance. J Cancer Res Clin Oncol 1998;124:421-426.

39 Shin KH, Park JG. Microsatellite instability is associated with genetic alteration but not with low levels of expression of the human mismatch repair proteins hMSH2 and hMLH1. Eur J Cancer 2000;36:925-931.

40 Kang YH, Bae SI, Kim WH. Comprehensive analysis of promoter methylation and altered expression of hMLH1 in gastric cancer cell lines with microsatellite instability. J Cancer Res Clin Oncol 2002;128:119-124.

41 Carethers JM, Hawn MT, Chauhan DP, et al. Competency in mismatch repair prohibits clonal expansion of cancer cells treated with $N$-methyl- $N^{\prime}$-nitro- $N$-nitrosoguanidine. J Clin Invest 1996;98:199-206.

42 Renault B, Calistri D, Buonsanti G, et al. Microsatellite instability and mutations of p53 and TGF-beta RII genes in gastric cancer. Hum Genet 1996;98:601-607. 